\title{
Correction to: A phase 2, open-label, multi-center study to evaluate the efficacy and safety of ${ }^{99 \mathrm{~m}}$ Tc-TRODAT-1 SPECT to detect Parkinson's disease
}

\author{
Yu Sun ${ }^{1}$. Congjin Liu ${ }^{1} \cdot$ Zhengping Chen $^{2} \cdot$ Biao $\mathrm{Li}^{3} \cdot$ Zhongwei Lv $^{4} \cdot$ Jingjing Lou $^{5} \cdot$ Jie Tang $^{2} \cdot$ Yuankai Wang ${ }^{1}$. \\ Guangming Zhang ${ }^{1} \cdot$ Xingdang Liu ${ }^{1}$
}

Published online: 10 January 2020

(c) The Japanese Society of Nuclear Medicine 2020

\section{Correction to: Annals of Nuclear Medicine https://doi.org/10.1007/s12149-019-01412-2}

The corresponding author of the article would like to remove "Jian Wang" in the author group. The revised author group is given in this correction.

Publisher's Note Springer Nature remains neutral with regard to jurisdictional claims in published maps and institutional affiliations.

The original article can be found online at https://doi.org/10.1007/ s12149-019-01412-2.

Jingjing Lou

1436150464@qq.com

$\triangle$ Xingdang Liu

xingdliu@fudan.edu.cn

1 Department of Nuclear Medicine, Huashan Hospital, Fudan University, No. 12 Wulumuqi Middle Road, Jing' an District, Shanghai 200040, China

2 Key Laboratory of Nuclear Medicine, Ministry of Health, Jiangsu Key Laboratory of Molecular Nuclear Medicine, No. 20 Qianrong Road, Binhu District, Wuxi 214063, Jiangsu, China
3 Department of Nuclear Medicine, Ruijin Hospital, Shanghai Jiaotong University, No. 197, Ruijin Er Road, Shanghai 200025, China

4 Department of Nuclear Medicine, Shanghai Tenth People's Hospital, Tongji University School of Medicine, 301 Yangchang Middle Road, Jing'an District, Shanghai 200072, China

5 Universal Medical Imaging Diagnostic Center, No. 406 Guilin Road, Xuhui District, Shanghai 200233, China 